\title{
Recent Results of Target Single-Spin Asymmetry Experiments at Jefferson Lab
}

\author{
Xiaodong Jiang ${ }^{* \dagger}$ \\ Los Alamos National Laboratory, Los Alamos, New Mexico, 87544. \\ E-mail: xjiang@bnl.gov
}

\begin{abstract}
We report recent results from Jefferson Lab Hall A "Neutron Transversity” experiment (E06-010). Transversely polarized target single-spin asymmetry $A_{U T}$ and beam-target double-spin asymmetry $A_{L T}$ have been measured in semi-inclusive deep-inelastic scattering (SIDIS) reactions on a polarized neutron $\left({ }^{3} \mathrm{He}\right)$ target. Collins-type and Sivers-type asymmetries have been extracted from $A_{U T}$ for charged pion SIDIS productions, which are sensitive to quark transversity and Sivers distributions, correspondingly. Double spin asymmetry $A_{L T}$ is sensitive to a specific quark transverse momentum dependent parton distribution (TMD), the so-called " transverse helicity" $\left(g_{1 T}\right)$ distributions. In addition, target single-spin asymmetries $A_{y}$ in inclusive electron scattering on a transversely polarized ${ }^{3} \mathrm{He}$ target in quasi-elastic and deep inelastic kinematics were also measured in Hall A.
\end{abstract}

The 7th International Workshop on Chiral Dynamics,

August 6 -10, 2012

Jefferson Lab, Newport News, Virginia, USA

\footnotetext{
* Speaker.

${ }^{\dagger}$ Work supported by U.S. Department of Energy, Office of Science.
} 


\section{Introduction}

Since the observation of surprisingly large single transverse spin asymmetries (SSA) in $p^{\uparrow}+$ $p \rightarrow \pi+X$ at Fermilab in the 1980s, the exploration of the physics behind the observed SSAs has become a very active branch in hadron physics. The field of transverse spin physics has now become one of the hot spots in high energy nuclear physics, generating tremendous excitement on both theoretical and experimental fronts.

Semi-Inclusive Deep-Inelastic Scattering (SIDIS) measurements on polarized targets have generated the first information on quark transverse spin (transversity) and transverse-momentum dependent parton distributions (TMDs), such as the quark Sivers distributions. The HERMES experiment at DESY carried out the first SSA measurement in SIDIS reaction on a transversely polarized proton target. The COMAPSS experiment at CERN carried out similar SSA measurements on transversely polarized deuteron and proton targets. Recently, Jefferson Lab Hall A published results of single spin and double-spin asymmetry measurements on a transversely polarized neutron $\left({ }^{3} \mathrm{He}\right)$ target $[1,2]$.

\section{Semi-Inclusive DIS results}

The angular dependence of the target single spin asymmetry $A_{U T}$ in the scattering of an unpolarized lepton beam off a transversely polarized target is:

$$
A_{U T}\left(\phi_{h}, \phi_{S}\right) \equiv \frac{1}{P_{T}} \frac{Y_{\phi_{h}, \phi_{S}}-Y_{\phi_{h}, \phi_{S}+\pi}}{Y_{\phi_{h}, \phi_{S}}+Y_{\phi_{h}, \phi_{S}+\pi}} \approx A_{C} \sin \left(\phi_{h}+\phi_{S}\right)+A_{S} \sin \left(\phi_{h}-\phi_{S}\right)
$$

where $P_{T}$ is the target polarization, $Y$ is the normalized yield. $A_{C}$ is the Collins moment, which arises from the convolution of the chiral-odd transversity distribution $h_{1}$ and the chiral-odd Collins fragmentation function (FF). $A_{S}$ is the Sivers moment, which arises from the convolution of the naive T-odd quark Sivers function $f_{1 T}^{\perp}$ and the unpolarized FF $D_{1}$. While the quark transversity distribution represents the probability of finding a transversely polarized quark inside a transversely polarized nucleon, the quark Sivers distribution reveals the correlation of the quark's orbital angular motion with the nucleon spin. Quark's Sivers distribution is transverse momentum dependent, and it is related to the imaginary piece of the interference between quark angular momentum $L=0$ and $L>0$ components of wave function, and is identically zero in the case that quarks carrie no angular momentum.

Another observable related to quark TMD is the longitudianally polarized beam double-spin asymmetry (DSA) $A_{L T}$ on a transversely polarized target:

$$
A_{L T}\left(\phi_{h}, \phi_{S}\right) \equiv \frac{1}{P_{B} P_{T}} \frac{Y^{+}\left(\phi_{h}, \phi_{S}\right)-Y^{-}\left(\phi_{h}, \phi_{S}\right)}{Y^{+}\left(\phi_{h}, \phi_{S}\right)+Y^{-}\left(\phi_{h}, \phi_{S}\right)} \approx A_{L T}^{\cos \left(\phi_{h}-\phi_{S}\right)} \cos \left(\phi_{h}-\phi_{S}\right)
$$

where $P_{B}$ is the polarization of the lepton beam. The $A_{L T}^{\cos \left(\phi_{h}-\phi_{S}\right)}$ moment is related to the convolution of quark's "transverse helicity" TMD $\left(g_{1 T}\right)$ and the unpolarized FF $D_{1}$. The TMD $g_{1 T}$ is corresponding to the real part of the interference between quark angular momentum $L=0$ and $L>0$ components of wave function, and it is identically zero when quarks carrie no angular momentum. In general, $g_{1 T}^{u}\left(g_{1 T}^{d}\right)$ is suggested to be positive (negative), and $g_{1 T}$ is shown to be closely connected with other TMDs through models. 
The E06-010 experiment [1, 2] was carried out in Jefferson Lab Hall A from Oct. 2008 to Feb. 2009. Measuring SSA and DSA in charged pion electro-production $n\left(e, e^{\prime} \pi^{ \pm}\right) X$ on a transversely polarized neutron $\left({ }^{3} \mathrm{He}\right)$ target is the main object of this experiment. The beam energy of the longitudinally polarized electron was $5.89 \mathrm{GeV}$. The average beam current was $12 \mu \mathrm{A}$ corresponding to a polarized luminosity of $\sim 10^{36} \mathrm{~cm}^{-2} \mathrm{~s}^{-1}$. The electron beam helicity was flipped at $30 \mathrm{~Hz}$ by changing the laser polarization with a Pockels cell at the injector. The fast beam helicity flip is a rather unique feature of the CEBAF machine, perfect in canceling out systematic uncertainties while forming $A_{L T}$ beam-helicity asymmetries. In comparison, at HERA, the electron (positron) beam helicity flips can only be achieved at a time scale of months by re-setting magnets in the machine, and COMPASS's muon beam from pion decays only carries one helicity state at a given beam energy. In E06010, beam polarization was periodically measured by a Mller polarimeter, and the average value is determined to be $(76.8 \pm 3.5) \%$. For SSA measurements, unpolarized beam was achieved by summing the two beam helicity states, with the residual beam charge asymmetry less than 100 ppm per 1-hour run.

A $40 \mathrm{~cm}$ long, 8 atms pressurized polarized ${ }^{3} \mathrm{He}$ cell was used which had an averaged polarization of $(55.4 \pm 2.8) \%$. The target polarization direction was controlled by three pairs of orthogonal Helmholtz coils. Two orientations, vertical and horizontal polarizations in the plane transverse to the beam direction was chosen to maximize the $\phi_{S}$ coverage in order to achieve a clear separation of Collins and Sivers asymmetries from the measured $A_{U T}$. The target spin was automatically flipped through the RF-induced Adiabatic Fast Passage (AFP) every 20 minutes regardless of other conditions. A reliable scheme of fast target spin flip, without altering the direction of the target holding field, is crucial in target SSA measurements to cancel out false asymmetries due to relative luminosity differences and possible drifts in detector responses corresponding to the two target spin states. Beam charge monitors upstream of the target provided integrated beam charge information corresponding to each target spin states. Single-spin asymmetries were formed based on accepted event counts, corrected by DAQ dead-time and beam charge difference between target spin-up and spin-down states.

Scattered electrons with momenta from 0.6-2.5 GeV/c were detected in the BigBite spectrometer at a central angle of $30^{\circ}$ on beam right. Coincident charged hadrons were detected in the High Resolution Spectrometer at a central angle of $16^{\circ}$ on beam left and a central momentum of 2.35 $\mathrm{GeV}$. Beyond the stage of raw data processing and particle ID cuts, two independent teams performed asymmetry analysis using completely different methods and software packages. One team extracted SSA by fitting in 2-D $\left(\phi_{h}, \phi_{S}\right)$ bins according to Eqn.2.1. The other team formed asymmetries by an un-binned maximum-likelihood method. Results were cross-checked at each major steps of the analysis to eliminate human errors and software bugs. The neutron SSA and DSA were extracted from the measured ${ }^{3} \mathrm{He}$ moments according to the effective nucleon polarizations in ${ }^{3} \mathrm{He}$.

The extracted neutron Collins and Sivers moments are shown in the Fig. 1. Collins moments are compared with the phenomenological fit [3], a light-cone quark model calculation and quarkdiquark model [6] calculations. Negative $\pi^{+}$Sivers moments are favored by these data, while the $\pi^{-}$moments are close to zero. Within the Leading-Order parton model interpretation, such behavior is consistent with a negative sign for the $d$ quark's Sivers distribution, which is consistent with the HERMES (proton) and the COMPASS (deutron and proton) data, a light-cone quark model calculation [4], and an axial diquark model calculation[7]. 


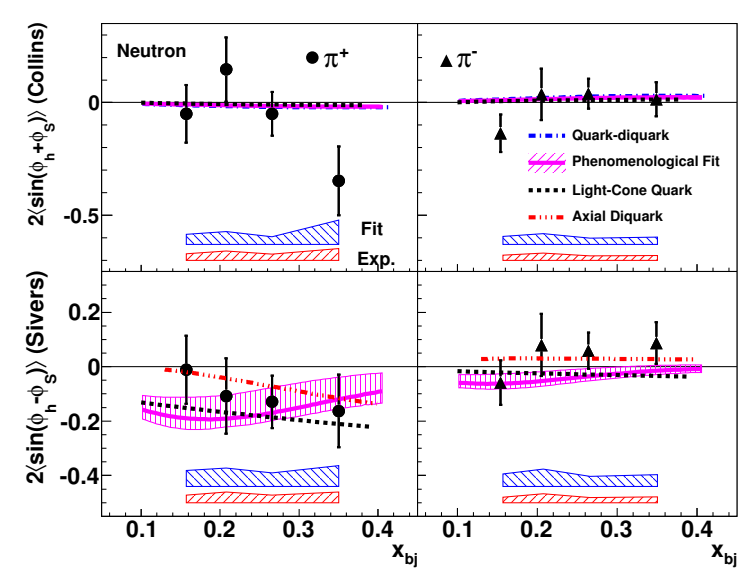

Figure 1: The extracted neutron Collins and Sivers moments with uncertainty bands from Ref. [1].

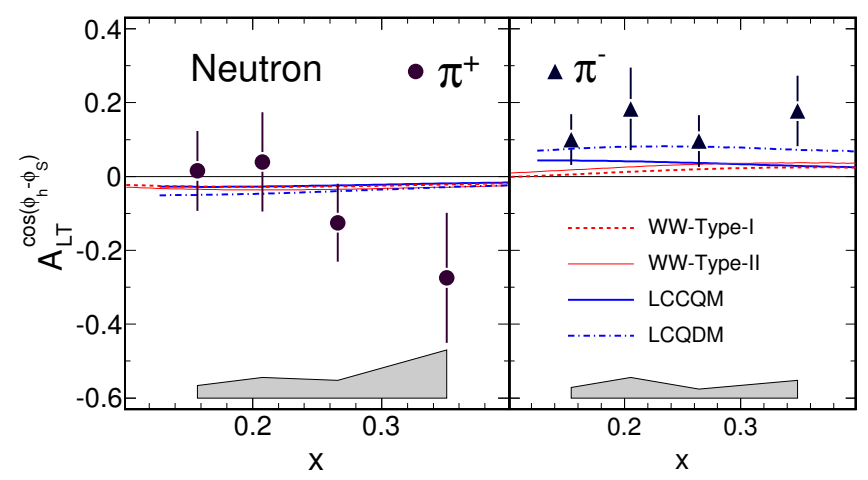

Figure 2: The neutron $A_{L T}^{\cos \left(\phi_{h}-\phi_{S}\right)}$ azimuthal asymmetry for positive (left) and negative (right) charged pions vs $x$ from Ref. [2].

The extracted neutron $A_{L T}$ moments are shown in Fig. 2. Several model calculations, including WW-type approximations with parametrizations Type-I from Ref. [8] and Type-II from Ref. [8], a light-cone constituent quark model (LCCQM) [4] and a light-cone quark-diquark model (LCDQM) evaluated using the second approach in the Ref. [9], are plotted as well. While the extracted $A_{L T}^{n}\left(\pi^{+}\right)$is consistent with zero within uncertainties, the results of $A_{L T}^{n}\left(\pi^{-}\right)$are consistent in sign with these model predictions but favor a larger magnitude. The sign of $A_{L T}^{n}\left(\pi^{-}\right)$is opposite to the sign of the $A_{U L}^{\sin 2 \phi_{h}}$ asymmetry in the $\pi^{+}$production on the proton measured by the CLAS collaboration.

\section{Inclusive channel single-spin asymmetry results}

In addition to SIDIS channels, SSA were also measured in inclusive hadron production ${ }^{3} \mathrm{He}\left(e, \pi^{ \pm}\right)$, ${ }^{3} \mathrm{He}\left(e, K^{ \pm}\right),{ }^{3} \mathrm{He}(e, p)$ and in inclusive electron scattering in DIS as well as in quasi-elastic ${ }^{3} \mathrm{He}\left(e, e^{\prime}\right)$ scattering. Since target in-plane SSA of inclusive channels are parity-forbidden due to $\left(\vec{k}_{1} \times \vec{k}_{2}\right)$. 
$\overrightarrow{S_{T}} \equiv 0$, measurements of SSAs in such a configuration would clearly demonstrate the control of false asymmetry. In JLab E06-010 experiment, ${ }^{3} \mathrm{He}$ SSA of all inclusive channels in target polarized in-plane configuration were well below $1.0 \times 10^{-3}$, demonstrated that false asymmetries were under control. The SSAs measured in target-vertical configuration were clearly non-zero for inclusive hadron production, at a few percent level with the same sign for $\pi^{+}, K^{+}$and proton, opposite to that of $\pi^{-}$. For inclusive electron channel at DIS kinematics, preliminary results indicated that SSA were less than the level of $1.0 \times 10^{-3}$. At quasi-elastic kinematics of $Q^{2}=0.1,0.5$ and 1.0 $\mathrm{GeV}^{2}$, non-zero SSAs signals at the level of a few parts $10^{-3}$ were clearly observed in inclusive ${ }^{3} \mathrm{He}\left(e, e^{\prime}\right)$ reaction. The final results of these inclusive channel SSA measurements will be submitted for publication soon.

\section{References}

[1] X. Qian et al. Phys. Rev. Lett. 107, 072003 (2011).

[2] J. Huang et al. Phys. Rev. Lett. 108, 052001 (2012).

[3] M. Anselmino et al., Phys. Rev. D75, 054032 (2007).

[4] S. Boffi et al., Phys. Rev. D79, 094012 (2009).

[5] B. Pasquini, S. Cazzaniga and S. Boff, Phys. Rev. D78, 034025 (2008).

[6] J. She et al., Phys. Rev. D83, 037502 (2011). B. Q. Ma et al., Phys. Rev. D65, 034010 (2002). B. Pasquini and P. Schweitzer, Phys. Rev. D83, 114044 (2011).

[7] L. P. Gamerg et al. Phys. Rev. D77, 094016 (2008).

[8] A. Kotzinian, B. Parsamyan, and A. Prokudin, Phys. Rev. D73, 114017 (2006)

[9] J. Zhu and B.-Q. Ma, Phys. Lett. B696, 246 (2011). 